\title{
Ultrafast pulse generation in integrated arrays of Anapole nanolasers
}

\author{
Juan Sebastian Totero Gongora ${ }^{1, *}$, Andrey E. Miroshnichenko², \\ Yuri S. Kivshar ${ }^{2}$ and Andrea Fratalocchi ${ }^{1}$ \\ ${ }^{1}$ PRIMALIGHT, King Abdullah University of Science and Technology (KAUST), Thuwal 23955-6900, Saudi Arabia \\ ${ }^{2}$ Nonlinear Physics Centre, Research School of Physics and Engineering, Australian National University, Canberra, ACT 2601, Australia
}

*Presenting author, js.totero@kaust.edu.sa, http://primalight.org

One of the main challenges in photonics is the integration of ultrafast coherent sources in silicon compatible platforms at the nanoscale [1]. Generally, the emission of ultra-short pulses is achieved by synchronizing the cavity modes of the system via external active components, such as, e.g., Q-switch or saturable absorbers. Consequently, the required optical setups are complex and difficult to integrate on-chip. To address these difficulties, we propose a novel type of integrated source based on the spontaneous synchronization of several near-field nanolasers. We design our near-field lasers by considering the nonlinear amplification of non-radiating Anapole modes [2]. Anapoles represent an intriguing non-conventional state of radiation, whose excitation is responsible for the formation of scattering suppression states in dielectric nanostructures [3]. Due to their inherent near-field emission properties, an ensemble of anapole-based nanolasers represent an ideal candidate to investigate and tailor spontaneous synchronization phenomena in a silicon-compatible framework. Additionally, their mutual non-linear interaction can be precisely controlled within standard nanofabrication tolerances.

We investigated the nonlinear emission from an ensemble of anapole-based nanolasers with our FiniteDifferences in Time- Domain simulator NANOCPP, which solves a dispersive set of 3D Maxwell-Bloch equations without approximation [5-9]. The single nanolasers were composed of three-dimensional III-V semiconductor nanostructures. By inducing the formation of a fundamental anapole state in correspondence of the emission frequency of the amplifying semiconductor, we obtained a lasing emission composed of purely evanescent waves (Fig. 1-a). By coupling several anapole sources the system behaves as an integrated ultrafast pulse generation device (Fig. 1-b), in which the collective emission is collected by a silicon waveguide. Interestingly, the system achieves a stable mode-locked emission with optical pulses of less than 100fs (Fig. 1c). The pulse duration and the repetition rate of the system can be tuned by varying the relative position of the anapole emitters, as shown in Fig. 1-d where the system is optimized to produce $p s$ optical pulses.
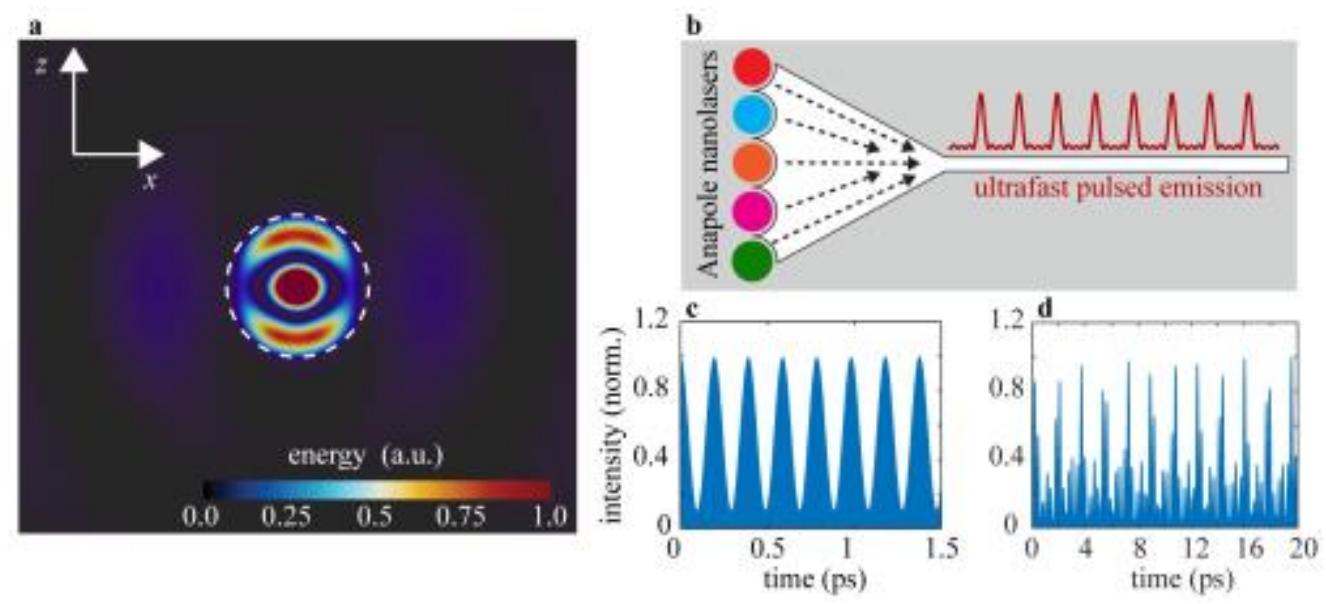

Fig. 1 Generation of ultrafast pulses from a chain of anapole-based nanolasers. (a) Near-field emission from a 3D anapole nanolaser. (b) Schematic representation of our ultrafast generation device. (c,d) Steady-state emission from the system. The pulse duration and repetition rate can be controlled by optimizing the geometry of the system.

\section{Example References}

[1] P. Miao et al., "Orbital angular momentum microlaser", Science 353, 6298, 464-467 (2016).

[2] A. E. Miroshnichenko et al., "Nonradiating anapole modes in dielectric nanoparticles", Nat. Commun. 6, 8069 (2015).

[3] W. Liu et al., "Invisible nanowires with interfering electric and toroidal dipoles,", Opt. Lett. 40, 2293 (2015).

[4] G. Grinblat, "Efficient THG and Nonlinear Subwavelength Imaging at a Higher-Order Anapole Mode in a Single Ge Nanodisk", ACS Nano, Article ASAP, DOI: 10.1021/acsnano.6b07568 (2016).

[5] C. Liu et al., "Triggering extreme events at the nanoscale in photonic seas", Nat. Phys. 11, 358-363 (2015).

[6] J. S. Totero Gongora et al., "Energy equipartition and unidirectional emission in a spaser nanolaser," Laser Photon. Rev. 10, 432 (2016).

[7] H. Galinski et al., "Scalable, ultra-resistant structural colors based on network metamaterials," Light Sci.App. 6, e16233 (2017).

[8] J. S. Totero Gongora and A. Fratalocchi, "Ab-initio techniques for light matter interaction at the nanoscale," in "Computational Chemistry Methodology in Structural Biology and Material Sciences”, ISBN: 9781771885683 (Apple Academic Press, Oakville, 2017).

[9] A. Fratalocchi et al., "Three-dimensional ab initio investigation of light-matter interaction in mie lasers," Phys. Rev. A 78 (2008). 